\title{
Reactivación vs turistificación: los retos de las ciudades frente al turismo post pandemia
}

\section{Recovery against touristification: Challenges of the cities facing post pandemic tourism}

Priscila Guerra Luzuriaga

ILAC International College, Canadá

Autor para correspondencia: prissyg194@ gmail.com

Fecha de recepción: 22 de septiembre de 2020 - Fecha de aceptación: 18 de diciembre de 2020

\section{Resumen}

El turismo se ha convertido en el mayor fenómeno social del mundo moderno, ha sido también el responsable de grandes transformaciones a nivel global, globalización y turismo han ido de la mano en los últimos años, sin embargo, el excesivo "éxito" del turismo, ha generado también grandes impactos. La gentrificación, la turistificación y más recientemente el overtourism, son efectos directos de lo que no se debe buscar cuando los países y regiones apuestan por el turismo como alternativa de desarrollo. La crisis sanitaria ocasionada por el COVID-19, mostró la fragilidad del sector turístico a escala global, siendo los grandes destinos turísticos los más afectados. Para el caso del Ecuador, al ser un destino emergente, también se ha visto afectado por la paralización total del sector turístico, frente a esta crisis el repensar que tipo de turismo deben plantearse las ciudades es clave. El presente trabajo parte de una revisión de literatura, que busca identificar la evolución de la turistificación en espacios históricos-patrimoniales, durante los últimos veinte años, al tratarse de una investigación de tipo exploratoria, con un enfoque cualitativo, se sustentó en un análisis histórico comparado, para ello, se analizaron destinos tanto de Latinoamérica como de Europa, en los cuales los efectos de la turistificación son por demás notorios y para el caso del Ecuador se tomó como referencia al barrio La Ronda en el Centro Histórico de Quito. Con los resultados obtenidos, se plantean una serie de orientaciones a partir de las cuales se debería repensar el proceso de reactivación turística, de manera particular aquellas ciudades en donde el impacto del turismo ha sido aún incipiente.

Palabras claves: turistificación; reactivación turística; ciudades; COVID-19.

\begin{abstract}
Tourism has turned out onto the greatest phenomenon on the modern world. It has been responsible of great transformations around the globe. Globalization and tourism have gone together during the last few years, nevertheless, tourism great success has resulted into considerable consequences. Gentrification, touristification and most recently, overtourism, are direct effects of what not to look for when countries and regions choose tourism as a development activity. The sanitary crisis that came with COVID-19, showed the fragile state of the touristic sector on a global scale, being the
\end{abstract}


greatest destinations the most affected ones. For Ecuador's case, being an emergent destination, it has also been affected due to total paralysation of the tourism industry. Facing this crisis, cities around the world must rethink the type of tourism they want to develop. The present academic work is based on a literature review, which aims to identify the evolution of touristification in patrimonial-historic spaces, on the span of the last 20 years. Since this is an exploratory study, with a qualitative focus, it was based on an historic-comparative analysis of Latin American and European destinations, where touristification is evident, while on Ecuador, La Ronda neighborhood on Quito's historic centre was taken as a reference. With the results obtained, a series of orientations were stablished, and sustained on them, the process of touristic reactivation must be reconsidered, particularly on cities where tourisms impact hasn't been significant.

Keywords: touristification; tourism reactivation; cities; COVID-19.

\section{Introducción}

El turismo, como actividad de significativa relevancia tanto económica como social se encuentra en constante transformación. En la actualidad la generación de nuevos patrones de consumo, encaminados a insertarse en el mercado utilizando como herramienta las nuevas tecnologías producto de la globalización (Molina, 2006) han resultado en el pos-turismo, caracterizado por propiciar ambientes que generen experiencias en determinados lugares, los cuales, a su vez se adapten a la individualidad de cada turista (Bezerra, Conceicao, y Rocha, 2012).

Por otra parte, el turismo urbano como modalidad turística que tiene lugar en las ciudades, específicamente en los "centros urbanos, y contempla la realización de diversas prácticas asociadas al ocio que realizan los turistas, durante su estancia, ya sean culturales, recreativas o profesionales" (Rodríguez, 2009, p,25) existente desde siglos precedentes, anclado al turismo cultural que a su vez, comprende el "movimiento de personas por motivos culturales como viajes de estudio, desplazamientos a festivales u otros eventos artísticos," (Organización Mundial del Turismo citado en Ayala, 2009, p.155), y que en la actualidad continúan presentándose como tipologías del turismo preferidas por los turistas en la actualidad (OMT, 2016), pero adaptado al ya mencionado pos-turismo. Es entonces que, dentro de las ciudades se definen los nuevos patrones en el paisaje urbano, y se introducen cambios en el mismo, con el fin de adaptarlo al consumo turístico. Es entonces que el turista internacional, responde a un turismo que se caracteriza por ser más heterogéneo, y especializado, lo que da como resultado la demanda de nuevas experiencias y el constante crecimiento de la actividad turística (Flores y De la O Barroso, 2012).

Por el contrario, cuando se produce el crecimiento desmedido y descontrolado de la actividad turística, se genera el fenómeno de la turistificación, que se define como el "impacto que tiene la masificación turística en el tejido comercial y social de determinados barrios o ciudades en que los servicios, instalaciones y comercios se conciben pensando solo en el turista" (Canalis, 2017), y cuyos efectos se observa claramente sobre todo en ciudades europeas como: Venecia, Madrid, Barcelona o Portugal; al tratarse de un concepto relativamente nuevo, los estudios académicos desarrollados al respecto se han suscitado de forma progresiva en años recientes. 
Por ende, el siguiente trabajo de investigación propone realizar una revisión de la literatura existente, para determinar la evolución del concepto de turistificación, y su influencia en espacios histórico-patrimoniales durante las últimas dos décadas. Para tal fin, se utilizó el método histórico comparativo de trabajos académicos relacionados a esta temática en particular, a su vez, la investigación se complementa con estudios de caso en ciudades de Europa y Latinoamérica, haciendo un particular énfasis en el histórico barrio de La Ronda de la ciudad de Quito en Ecuador, y en base a los resultados obtenidos se busca determinar orientaciones que permitan delinear algunas estrategias que den respuesta al decaimiento de la actividad turística en la etapa post COVID-19.

\section{Turistificación y Gentrificación: Su influencia en espacios históricos -patrimoniales}

Para comprender a cabalidad las diferencias existentes entre ambos términos, y la manera en que influyen en los espacios histórico-patrimoniales de las ciudades, se tomó en cuenta las siguientes conceptualizaciones: En primera instancia, se define a los espacios históricos patrimoniales como "espacios urbanos que comprenden un patrimonio físico, urbano y arquitectónico, en una zona céntrica de la ciudad y que representan los valores simbólicos identitarios de una sociedad" (Rodríguez, 2008, p.25). Complementariamente, Soto (2016) se refiere a ellos como el "bien nacional de uso público, destinado a la circulación y el esparcimiento que posee de manera intrínseca un valor patrimonial histórico, cultural y/o paisajístico" (Soto, 2016, p.37). Es en resumen "la materialización de la memoria histórica de una ciudad, en los que se desarrolla colectivamente la cultura, representados por un valor patrimonial de uso social” (Cantero, Escalera, García del Villar, y Hernández, 2000, p.127).

También cabe agregar que los espacios históricos - patrimoniales, se encuentran por lo general en localizaciones céntricas de las ciudades, y son también reconocidos en los centros históricos, definidos como áreas de "valor cultural y arquitectónico que forma parte de un espacio metropolitano o ciudad de considerable población, y que posee complejas y diversificadas funciones, además de una densidad demográfica importante" (Hardoy 2002, citado en Chateloin, 2008, p.10), por lo que se vuelven el punto focal de la ciudad y un lugar de encuentro de las sociedades que los conforman (Carrión, 2005).

Teniendo en cuenta las definiciones previas, se debe tomar en consideración que como hecho histórico el cambio de actividades económicas en los centros históricos de las ciudades y el abandono del inmobiliario patrimonial en estos lugares, sumado a la privatización de espacios urbanos por parte de las administraciones públicas, destinados a ser restaurados, resultaron en la gentrificación, que se define como la "reocupación de un espacio urbano por parte de una clase socioeconómica en detrimento de otra, siendo esta última excluida mediante la diversificación forzada, por los mecanismos de mercado del precio de bienes raíces” (Checa, 2010, p.14). Es decir, se reemplaza a los antiguos habitantes de un barrio o sector determinado, por otros con mayor poder adquisitivo. La gentrificación turística, no es sino un fenómeno similar, en que, en lugar de ser reemplazados por otra clase social, los residentes habituales son desplazados por turistas.

Por otro lado, la turistificación es un concepto reciente, y para entender cómo se desenvuelve, se toma como antecedente ciertos aspectos. El turismo urbano en espacios 
históricos patrimoniales se ve confrontado con el rediseño de los espacios urbanos, adecuados a las necesidades de los turistas, es decir, que, de acuerdo Hiernaux y González (2015), es impulsado por medio de programas de apoyo a su exclusivo desarrollo, para recuperar los centros históricos, resignificando los espacios en pos del turismo, el ocio y el comercio. Esto suscita a su vez, en un cambio en las zonas urbanas, para potenciar el creciente negocio turístico (Cabrales, 2005; Crossa, 2009, citados en Janoschka, Sequera, y Salinas, 2014).

Es así que los espacios urbanos, y los sitios históricos - patrimoniales se modifican "la ocupación progresiva de hoteles y equipamientos para visitantes y una polarización creciente de flujos turísticos" (Navarrete, 2017, p.64) convierte, por tanto, al turismo en la principal fuerza económica, social y cultural, para la transformación de estilos de vida locales (Xian Liang y Gang Bao, 2014), que sumado al turismo de masas en ciertos destinos, permite que se genere el proceso de turistificación, que yendo más allá de la gentrificación habitual, o turística, no solo ocupa espacios urbanos, sino que se extiende hacia la completa transformación de la cultura y forma de vida de las ciudades y sus habitantes.

Cabe mencionar también que para que se susciten procesos de turistificación, afirman Crespi y Mascarilla (2018) “se requieren procesos de transformación urbana, desarrollados desde la administración local, en busca de la rehabilitación y mejora pública de espacios e infraestructuras, en aras al desarrollo del potencial turístico" (Crespi y Mascarilla, 2018, p.63). Esto implica que los entes gubernamentales, son los propiciadores de la turistificación en espacios urbanos. Al respecto, Onghena y Milano (2015), lo definen acertadamente como "el proceso a través del cual, una realidad histórica, social o cultural, se vuelve un valor en el mercado turístico, lo que permite su comercialización (...) es una oferta que se vuelve exótica" (Onghena y Milano, 2015, p.3).

Todo lo previamente mencionado se complementa, finalmente con un nuevo concepto conocido como overtourism como consecuencia de la turistificacion, que a su vez, de acuerdo a The Ostelea School of Tourism and Hospitality (2017) también degenera en la privatización del espacio público y la congestión de este en las ciudades, el aumento de los precios residenciales, y finalmente la pérdida del poder adquisitivo por parte de los residentes en los destinos, quienes a su vez, se han visto forzados a cambiar sus estilos de vida, y a desplazarse fuera de los centros históricos, producto de los elevados costos de vida, adaptados a los turistas (Novelli, 2018).

Y es entonces que, sobre todo en ciudades de Europa, la turismofobia se origina entre los habitantes como "una respuesta de fortalecimiento de la comunidad local ante el desplazamiento espacial ocasionado por el turismo" (Sequera y Nofre, 2018), se manifiesta de forma pública de acuerdo a Milano (2018), mediante movilizaciones de distintos movimientos sociales, representantes de la sociedad civil, quienes denuncian los efectos negativos del turismo en las ciudades, como; "la expulsión de los residentes, el aumento de la inseguridad y pérdida de la calidad de vida, y la transformación de los rasgos identitarios de los espacios urbanos” (Huete y Mantecón, 2018). 


\section{Materiales y métodos}

Una vez establecida la base teórica en la que se sustenta la investigación, se determina la metodología utilizada para le elaboración del presente estudio. El cuál parte de una investigación con un enfoque cualitativo, ya que se analizaron los hechos del fenómeno de estudio, en particular la turistificación, sus antecedentes históricos, así como las medidas de reactivación turística orientadas a la etapa post COVID-19. Posteriormente se desarrollaron perspectivas teóricas en base a la recopilación de datos de fuentes bibliográficas (Hernández Sampieri, Fernández Collado, y Baptista Lucio, 2014)

La tipología utilizada, fue de carácter exploratorio, lo que implicó "examinar o explorar un tema o problema de investigación poco estudiado que no ha sido abordado antes (...)" (Cazau, 2006), que es el caso de la turistificación, ya que, al tratarse de un neologismo, aún se trata de un concepto en construcción. Así también, referente a las acciones realizadas para la reactivación del turismo, debido a que se trata de un acontecimiento aún en desarrollo, en respuesta a la pandemia.

Los métodos utilizados fueron, el histórico comparado, que a través del análisis documental enlaza los elementos comunes en un hecho histórico, en relación con la turistificación, y su influencia en los destinos actuales, y las acciones orientadas la reactivación post COVID-19 (Tardivo y Fernández, 2017). Por otro lado, se ha incluido también el analítico sintético, que comprende "la descomposición del objeto de estudio en sus distintos elementos o partes componentes para obtener nuevos conocimientos acerca de dicho objeto" (Hurtado León y Toro Garrido, 2007) y, por ende, permitió establecer la base para la construcción del marco teórico en el que se sustentó la presente investigación, así como la generación de sus respectivos resultados.

Respecto a las técnicas de investigación usadas, el estado del arte fue una de ellas "Es una investigación documental sobre la cual se recupera (...) "el conocimiento acumulado sobre determinado objeto de estudio, cuyo fin es develar la interpretación de autores sobre el fenómeno y hacer explicita la postura teórica y metodológica de diferentes estudios" (Galeano Marín y Vélez Restrepo, 2002, Citado en Gómez, Galeano, y Jaramillo, 2015) y mediante la cual se recolectó el material necesario para desarrollar los conceptos claves de la presente investigación.

Finalmente, se aplicó también el estudio de caso, con el cual se buscó realizar una caracterización de aquellos destinos que han sido considerados como territorios turistificados o que por su condición turística han tenido algún nivel de gentrificación turística, destacándose: Madrid, Porto, México, Cartagena y Quito (La Ronda). La recolección de los datos ha sido obtenida desde diversas fuentes bibliográficas, siendo estos; documentos, archivos, artículos científicos, así como, medios electrónicos y diversas páginas web- Chetty (1996) citado en Martínez (2006). 


\section{Resultados}

Para determinar el proceso de evolución de la turistificación, se realizó un análisis de fuentes académicas de los últimos 20 años, como se muestra en la figura 1, las cuales dieron paso a la base teórica de la investigación.

\section{Figura 1}

\section{Fuentes bibliográficas consultadas}

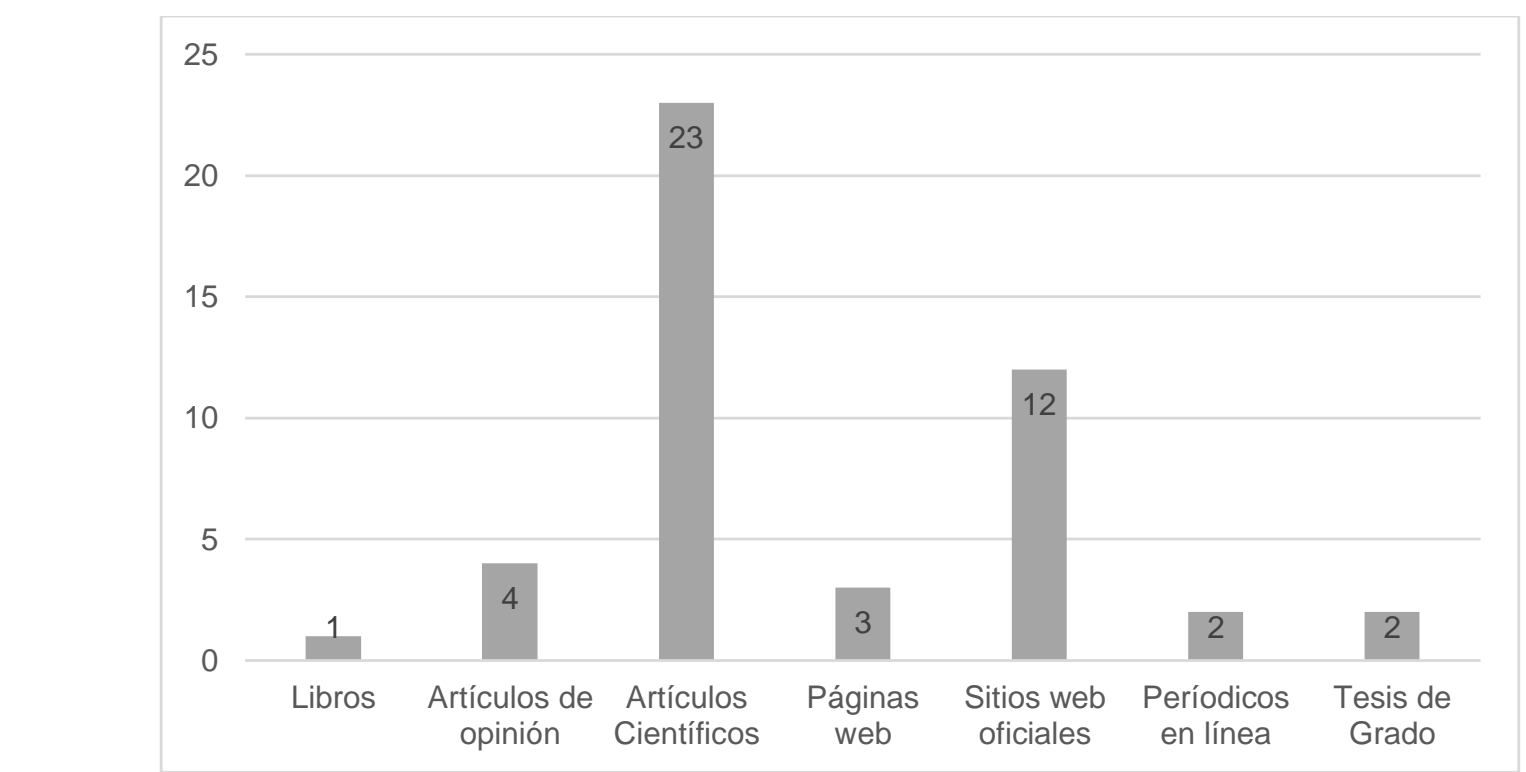

Elaboración propia

Se obtuvieron un total de cuarenta y siete fuentes bibliográficas durante el levantamiento de la información para la posterior construcción del estado del arte. Del total, veintitrés, constando como la mayoría, fueron artículos científicos, ya que el fenómeno de la turistificación es un concepto relativamente reciente. Mientras que las fuentes restantes, cuentan con un número considerablemente menor. Los sitios web oficiales, a los que corresponde un total de doce fuentes consultadas, se refieren a las páginas correspondientes a entidades de turismo, considerando a la OMT, así como, a cada una de las organizaciones que representan a los países previamente mencionados. Las fuentes restantes representan un número considerablemente menor.

Por otra parte, también se obtuvieron conceptos clave para el desenvolvimiento de la base teórica investigativa, los cuales se presentan a continuación, acompañados de los autores que se consideraron más relevantes: 


\section{Tabla 1}

Conceptos claves para el desarrollo de la investigación

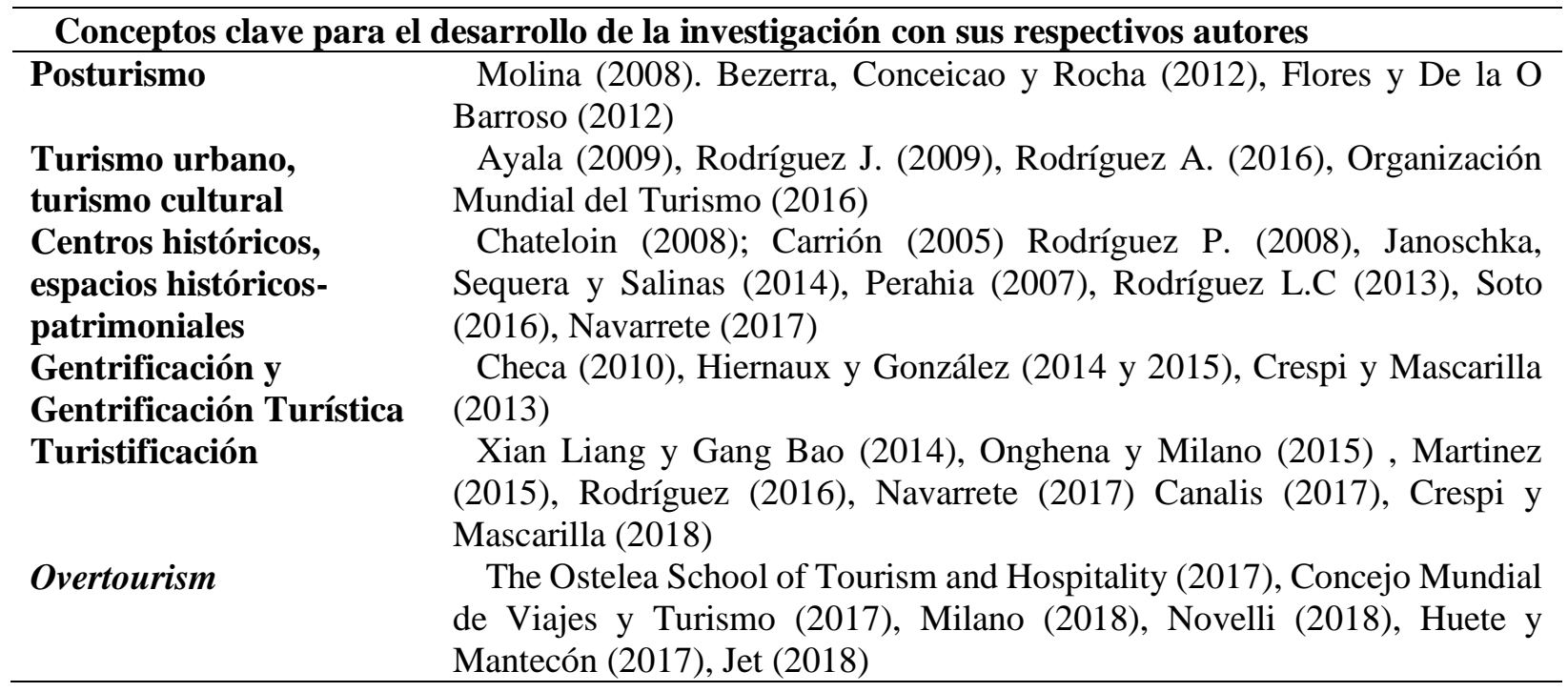

Elaboración propia

Se obtuvieron un total de 27 autores, basándose en nueve conceptos clave, relacionados con: posturismo (3), turismo urbano y cultural, centros históricos (4), espacios históricospatrimoniales (9), gentrificación, gentrificación turística (4), turistificación (6) y Overtourism (6), elementos abordados, previamente en la introducción y marco teórico del presente artículo.

\section{Estudios de caso a nivel internacional}

A partir de las fuentes bibliográficas documentadas se obtuvieron datos respecto al proceso de turistificación en los siguientes destinos:

El turismo en España presenta efectos positivos en su economía, a su vez permite la difusión de su cultura y la dinamización de la vida urbana en sus ciudades. Sin embargo, también conlleva efectos negativos, en ciudades como Barcelona, en donde la actividad turística se dirige a numerosos grupos especialmente de jóvenes, cuya principal motivación es un turismo nocturno, lo que ha provocado un efecto negativo en los residentes, dada la contaminación acústica y la desorganización que ha provocado un efecto en lugares como Citat Vella y las Ramblas en mayor medida (Crespi y Domínguez, 2013). En Madrid, se observa un fenómeno similar, ya que el uso de suelo ha sido destinado en su mayoría a bares, hoteles, galerías de arte y pisos de alquiler. Los habitantes denuncian contaminación, exceso de personas en los espacios, sensación generalizada de inseguridad, entre otros, por lo que los turistas no son capaces de coexistir con la forma de vida tradicional del barrio.

Porto, en Portugal, se ha convertido en un popular destino europeo. La influencia de la actividad turística lo ha transformado "en un destino turístico global, principalmente por la 
promoción cultural de la ciudad mediante el uso intensivo de técnicas de marketing urbano, y la mejora de las conexiones aeroportuarias" (Rodríguez, 2016, p.6). EL turismo ha transformado los espacios, adaptándolo a turistas con gran poder adquisitivo, por lo que se ha generado disconformidad en la población debido al elevado precio de la vivienda y los servicios complementarios (ocio, bienes de consumo).

El Centro Histórico de Querétaro en México, se ha visto afectado en el área central, por el creciente interés en las actividades turísticas por instalar en las áreas patrimoniales y las rentas que se pueden percibir de parte de ellas, siendo así, "buena parte del Centro Histórico se ha visto conquistado por la presencia de Hoteles Boutique, restaurantes y tiendas ligadas al ocio y al turismo" (Hiernaux y González, 2014, p.7). Se han cerrado negocios tradicionales, y se han adaptado las políticas de uso de suelo para la construcción de sitios designados a negocios relacionados el turismo, produciendo la movilización de los residentes habituales.

Al ser declarada Patrimonio de la Humanidad, Cartagena de Indias atrajo a los inversionistas del ámbito privado, quienes adquirieron bienes residenciales para su posterior renovación, por lo que se suscitó el alza de los costos inmobiliarios, siendo Getsemaní el barrio que más se ha considerado afectado recientemente, reduciéndose su población original a un $28 \%$ del total de residentes actuales. Al ser dominado el paisaje urbano por hoteles de alta categoría y restaurantes o bares, Getsemaní quedó en manos de agentes económicos y turísticos externos (Rius-Ulldemolins y Posso, 2016). Por lo cual, las agrupaciones civiles y culturales, como las asociaciones de vecinos, y la junta de Acción Comunal surgen para reivindicar la identidad tradicional promoviendo iniciativas para generar consciencia en los habitantes de la gravedad del fenómeno, como una respuesta reaccionaria al turismo.

Un punto en común en los destinos latinoamericanos es que los procesos de turistificación se suscitan en centros históricos, mediante la regeneración urbana de los mismos, por parte de entidades gubernamentales y posteriormente aceptar inversiones provenientes del sector privado, en forma de hoteles, restaurantes, tiendas, entre otros. En un principio se puede presentar como un beneficio económico para los residentes, sin embargo, a la larga, la dinámica existente en los barrios se ve mermada, por el alza de precios continua, el cambio de la cultura local, la llegada de nuevos habitantes que no se adaptan a los habitantes originales, razón por la que el estilo de vida de los habitantes se ve afectado (Guerra Luzuriaga, 2019).

\section{Ecuador - Estudio de Caso La Ronda}

La ciudad de Quito fue declarada Patrimonio Cultural de la Humanidad por la UNESCO en el año de 1978, por lo que se han desarrollado políticas de regeneración urbana en pos de la mejora de la ciudad en beneficio de la ciudadanía (Salgado, 2008) recuperando los espacios públicos y renovando los bienes patrimoniales, además se da también el desarrollo de locales comerciales (hoteles, restaurantes) con fines turísticos a forma de un beneficio adicional.

Este el caso del Barrio La Ronda, el cual, fue renovado en el año 2006, por parte del FONSAL ante la aprobación de gran parte de la ciudad, mientras que varios de sus otrora sitios residenciales, se destinaron posteriormente a actividades comerciales o en su mayoría turísticas. El proyecto de regeneración estuvo orientado entonces a "reforzar el carácter patrimonial del 
lugar, pero para determinadas élites, dejando de lado las prácticas y dinámicas habituales que habitaban el lugar" (Durán, 2014, p. 31)

En el año 2018, se registró una entrada de 693.076 turistas en la Ciudad de Quito, de los cuales un $67 \%$, visitó el centro histórico en su conjunto; mientras que un 9,22\% del total, consideró al barrio La Ronda como su lugar de visita (Quito Turismo, 2018)

Desde un punto de vista económico, la actividad turística no es significativa, "no existe una satisfacción general con las actividades que se realizan en la zona, ya que el $67 \%$ de la población considera que no perciben un beneficio directo del turismo" (Cazar, 2017, p. 41).

Hoy en día, existen "90 negocios, 56 casas patrimoniales, bohemia, vecindad e historia que forman parte de la calle la Ronda, por lo que en su vasta mayoría ya no está destinada a fines residenciales" (Guerrero, 2018), por lo que de parte de los moradores restantes en la zona, la disconformidad con la propagación de la actividad turística de la Ronda también es evidente desde distintas aristas, ya que algunos de los comerciantes no se atienen al uso de suelo destinado en principio a servicios culturales, sino que están enfocan en un turismo de vida nocturna, lo que resulta en un problema considerable en el incremento de la inseguridad generalizada(Castillo, 2012). Esto también responde al fenómeno mencionado por Sequera y Nofre (2018) como turistificación de la noche urbana, que envuelve el deterioro de una comunidad, dada la expansión de las facilidades orientadas al turismo, que permiten prosperar la vida nocturna y con ello el incremento de alcoholismo, desorganización, delincuencia, y contaminación acústica. Existe por tanto un proceso de turistificación en la Ronda, ya que la reacción negativa al turismo, no depende solamente del gran número de personas en un destino sino también de la saturación del mimo, percibida por los habitantes del mismo, ya que ven perjudicados sus espacios públicos y su bienestar local, por la presencia del turismo (Milano, 2018).

\section{Discusión}

\section{El COVID-19 y su efecto sobre los destinos turísticos}

Originalmente la Organización Mundial del Turismo tenía previsto un crecimiento del 4\% de la actividad turística, en relación con el año 2019, que fue de 1500 millones de llegadas a nivel global. El turismo entonces estaba confirmado como un sector en crecimiento y resiliente, por lo que se llamaba a un crecimiento responsable, para aprovechar las oportunidades que este traería consigo para los distintos destinos alrededor del mundo. (World Tourism Organization, 2020a)

Sin embargo, con la llegada del COVID-2019 alrededor del mundo, dadas las condiciones que permitieron su vertiginosa expansión, desde Wuhan, a finales del 2019 hasta inicios de 2020, producto del "Gran Festival de la Familia" o "Wang Jia Yan" en que más de 40 mil familias se desplazaran a este destino, y posteriormente se movilizaran alrededor de todo el país y a destinos alrededor del mundo. (Frutos, Lopez -Roig, Serra Cobo y Devaux, 2020) 
Para el siete de mayo del presente año, el turismo internacional se contrae un $22 \%$ en el primer trimestre y si bien se estimaba que decaería entre un 60 a $80 \%$ en el conjunto en el año (World Tourism Organization,2020b), la pérdida resultó ser mucho mayor, para el tercer trimestre del presente año, se calculan de "850 millones a 1100 millones de pérdidas en llegadas de turistas internacionales y de 910 mil millones a 1200 millones de pérdidas económicas, así también se estimó que alrededor de 100 millones de tasas de empleo se encontraban en riesgo" (Consejo Ejecutivo 112 de la OMT, tomado de Hosteltur, 2020), por lo que la recuperación de esta actividad estimaba su recuperación parcial hasta el primer trimestre de 2021.

\section{En camino a la reactivación del turismo}

En base a la complicada situación, la Organización Mundial del Turismo, decide poner en marcha el Plan de Recuperación del Turismo, para Apoyar Trabajos y economías a través del turismo Un llamado a la acción para mitigar los impactos socio económicos del COVID-19 y acelerar la recuperación (World Tourism Organization, 2020c), basado en tres pilares fundamentales, y sus respectivos subtemas, que se presentan a continuación de forma sintetizada:

Para empezar, se enfoca en la recuperación económica, para medir los impactos del COVID-19, mediante análisis cualitativos y cuantitativos de la situación actual, además de desarrollar planes de recuperación estratégicos en cada país de acuerdo a su respectivo contexto, y, asimismo, el apoyo a programas de micro, pequeñas y medianas empresas, con el fin de reorientar la cadena de valor del turismo hacia la sostenibilidad.

El segundo pilar es marketing y promoción, y se sustenta en hacer crecer escenarios potenciales que permitan potenciar los mercados existentes, complementando con una estrategia de marketing dirigida hacia mercados internacionales y nacionales, además de otra estrategia de marketing focalizada en mercados temáticos o regionales, que permitan diversificar el turismo, mediante el uso de marketing digital, sumado a una serie de incentivos para estimular el deseo de viajar al destino.

Por último, el fortalecimiento de instituciones y construcción de resiliencia, estrategia enfocada en las empresas para que estas se adapten a la realidad del COVID-19 manteniendo los estándares de seguridad e higiene. Se enfatiza también en el desarrollo de alianzas público privadas para la recuperación del turismo, así como la formulación de estrategias de comunicación durante la crisis, el crecimiento de sectores estratégicos, la creación de programas de capacitación para desarrollo turístico, el desenvolvimiento de estrategias para el desarrollo del capital humano, el fortalecimiento de las organizaciones de gestión de destinos, en complemento con programas de capacitación para comunidades locales que permitan de la creación de productos locales. Además, se hace alusión al empoderamiento de las mujeres a través del turismo, junto con un proyecto que incentive el empleo joven en el sector turístico (UNWTO, 2020).

Entre todos estos principios, algunos de los destinos mencionados previamente, en el marco teórico, otrora afectados por la turistificación, y ahora por la crisis post-pandemia, han implementado distintas iniciativas en sus territorios, con el fin de reactivar la actividad turística: 
En España por ejemplo tienen claro que a corto plazo la solución es el turismo doméstico, las capacidades de carga en destinos como Barcelona o Madrid se verán reducidas, plataformas en ciertos sitios como Airbnb, serán usadas en algunos de los destinos, por el hecho de no ser compartidos con otras personas y representar una idea generalizada de seguridad. La idea de turismo sostenible se fortalecerá, y cada destino del país garantizar medidas de bioseguridad para asegurar una visita tranquila y sin imprevistos a los turistas (Such Devessa, Ramon y Aranda, 2020). Así también el gobierno ha planteado un Plan de Impulso del Sector Turístico, dirigido hacia un turismo sostenible post COVID-19, basado también en una campaña de branding y promoción para mejorar la competitividad del destino en el mercado internacional (Portal Oficial de Turismo de España, 2020). También se plantearon planes de reactivación del turismo tanto en Barcelona como en Madrid, con miras hacia el primer trimestre del 2021 (La Moncloa, 2020). Todas las iniciativas previamente mencionadas se corresponden con los principios planteados por la OMT ya resumidos en el Plan de Reactivación Turística, en específico el pilar correspondiente al marketing y promoción, además del desarrollo de planes de recuperación estratégicos en los destinos.

Desde otra perspectiva, en Colombia se lanza un plan para reactivar el turismo desde el mes de Agosto, en el que se plantean la extrema relevancia en mantener las medidas de bio seguridad en establecimientos y atracciones para preservar la salud y seguridad de turistas y residentes, así como la apertura de 16 aeropuertos para el desplazamiento de turistas nacionales para empezar, y las playas alrededor de la costa del país, con el fin de incentivar la movilización de visitantes (Cantillo, 2020). Entretanto se destaca el esfuerzo de Cartagena de Indias, quienes, durante este tiempo, focalizaron sus esfuerzos para la creación del plan de desarrollo de Cartagena 2020 -2023, que ya se encuentra en línea. Entre las estrategias planteadas en el mismo se encuentran el fortalecer el talento humano asociado al sector turístico en la ciudad, el fortalecimiento socio económico, la diversificación de los productos existentes, mejorar la capacidad instalada, en relación con infraestructura existente, y en base a ello producir estrategias de comunicación para dar a conocer el destino. Así mismo, se enfatiza en realizar turismo con conciencia social y sentido, para generar entornos seguros y proteger al turista (Corporación de Turismo de Cartagena de Indias, 2020). Colombia también se encuentra encaminada en base al Plan de Reactivación Turística de la OMT, específicamente en el último pilar, destacando el fortalecimiento de instituciones, el desarrollo de planes estratégicos para su desarrollo, y el fortalecimiento de quienes trabajan en el sector.

Por otra parte, en México, desde el gobierno central se establece una guía para el turista internacional que opera con un semáforo de riesgo epidemiológico, con el fin de permitirle saber al turista, sobre todo que se desplace internamente, cuáles son los riesgos en los distintos destinos (Gobierno de México, 2020). Naturalmente y en comparación a otros destinos, también se establecen medidas de seguridad, con el respectivo plan de reactivación económica para el turismo nacional, mediante el fortalecimiento de pequeñas y medianas empresas de turismo, con incentivos fiscales y económicos y proponiendo un Fondo de Rescate para pequeñas y medianas empresas (Aguirre, 2020). En complemento se propone también relanzar el programa de pueblos mágicos en cuanto sea posible, así como la creación de un Consejo de Promoción para el Turismo Sustentable (Mena y Tlaxcala, 2020). También se alinea con los principios propuestos por la OMT, en el fortalecimiento económico de pequeñas y medianas empresas, así como el desarrollo de estrategias locales con gobiernos locales. 
En Ecuador por otra parte, el escenario se desenvuelve desde un panorama negativo en que el "sector turístico estima que perderá mensualmente hasta unos USD 400 millones a causa de la pandemia que obligó al cierre de fronteras en el país” (El Comercio, 2020)

Es así que el Ministerio de Turismo ha planteado medidas como la iniciativa " Te prometo Ecuador" así como el establecimiento de protocolos de bioseguridad en establecimientos de alojamiento, alimentos y bebidas y agencias de viaje, y el reciente reconocimiento a la capital como destino seguro de parte del Ministerio de Turismo (Ministerio de Turismo -MINTUR, 2020) Complementariamente el Distrito Metropolitano de Quito crea un Plan Emergente para la Reactivación del sector turístico, que plantea nuevas perspectivas y orientaciones para el sector, estableciendo principios para su recuperación mismo que se estructura en ocho estrategias clave, entre las cuales se encuentran: El reestructurar mercados que sean prioritarios, el identificar tecnologías y nuevas tendencias, contactar con nuevos actores, priorizar la seguridad turística, reconocimiento a la calidad, mantener los protocolos de bioseguridad y en base a ello, realizar las capacitaciones necesarias, así como la asistencia requerida para la recuperación paulatina de empresas turísticas y finalmente la articulación con empresas(Quito Turismo,2020). Estos planes también se alinean con las directrices de la OMT, en el pilar dedicado a la recuperación económica, enfatizando en la recuperación de mercado y apoyo a empresas turísticas, así como en el tercer pilar anclado a la resiliencia y fortalecimiento de instituciones ya que se busca nuevas alianzas estratégicas, así como el incentivo a las capacitaciones con el fin de adaptarse a la nueva realidad presente en el sector.

Todos estos planteamientos son aún recientes, por lo que queda esperar a observar su desenvolvimiento en un entorno cambiante, donde el COVID-19 será una constante en la vida común, y, por ende, las actividades recreativas tomarán un tiempo considerable en recuperarse, sin embargo, las iniciativas mencionadas responden a la guía establecida por la OMT, tanto como las necesidades de cada nación en particular, por lo que resta esperar a la posible recuperación del sector turístico el año próximo.

\section{En camino hacia la recuperación del turismo con miras a evitar la turistificación}

La turistificacion y la precarización de los espacios a favor de la actividad turística, crearon efectos negativos en los destinos turísticos, demostrados en una clara oposición al mismo de parte de la población local. Superada una vez esta época, y tras una pronunciada crisis producto de la pandemia queda como alternativa clara, replantear la actividad turística de sde una perspectiva más justa y sostenible con los habitantes locales y en los espacios en que se desarrolla la actividad turística. El ya mencionado overtourism dará paso a destinos sostenibles, es la única manera en que el turismo puede reinventarse. La afluencia de turistas deberá verse controlada para sobrellevar el temor que quedará como estelas de la crisis post pandemia (El Salto, 2020).

Sin embargo, cabe recalcar, que aún está por verse que tan efectivos serán los planteamientos señalados y si en base a ello el turismo en estos países podrá recuperarse de forma gradual hasta el tercer trimestre del 2021 que son las fechas estimadas que se toman como referencia. Si bien es cierto que las iniciativas generadas por los gobiernos se adhieren a varias de las directrices estipuladas por la OMT, no se tiene certeza de la manera en que las medidas del 
distanciamiento social y restricciones de movilidad afectarán a la llegada de turistas, sobre todo en el ámbito internacional.

\section{Conclusiones}

La actividad turística es un enclave económico necesario para el desarrollo de los destinos a nivel mundial. Sin embargo, el surgimiento de las movilizaciones masivas de turistas hacia los destinos urbanos, además de la influencia negativa de los mismos en la cultura local, dan como resultado el fenómeno de la turistificación, neologismo que surge como respuesta a la masificación del turismo y que al suscitarse en espacios histórico patrimoniales y centros históricos de una ciudad determinada sin el debido control, concurren en la resistencia de la población al impulso turístico en una determinada localidad.

Es entonces importante resaltar, que no solo la escasa planificación turística, sino también la masificación de los destinos, fueron hechos concretos para que el COVID-19 se difundiera con mayor rapidez. La expansión radicó en el escaso y pobre control de los destinos a la entrada de turistas, incluso con las numerosas advertencias provenientes de China, una vez que el virus empezó a propagarse a nivel mundial.

El superar las secuelas que ha dejado tras de sí el COVID-19, no solo de índole económico, sino también social y psicológico, será prioritario, por lo que el plano turístico se verá relegado hasta entonces, y por esta misma razón se procurará consolidar estrategias, a largo plazo que otorguen seguridad al turista. El turismo es una actividad económica de gran relevancia, por esta razón su planificación es clave para su desarrollo en beneficio de toda la población

La situación actual, por tanto, dará paso a destinos sostenibles. La afluencia de turistas deberá verse controlado para sobrellevar el control del destino y procurar que se cumplan con las medidas de bioseguridad, para mantener el orden, y preservar la integridad de los ciudadanos y ciudadanas de un determinado país. En el caso de los centros históricos, volver a preservarlos, además de precautelar la seguridad no solo del turismo, sino de la salud y sobre todo de los residentes habituales será de vital importancia. La puesta en acción de los planes y la formación de alianzas estratégicas son clave para la reactivación de la actividad turística. Ya teniendo en cuenta los antecedentes que llevaron a la complicada crisis que atraviesa el sector, se debe tomar en cuenta los errores del pasado para no volver a repetirlos.

\section{Referencias bibliográficas}

Aguirre, J. (junio de 2020) Caída del turismo por la COVID-19. Desafío para México y experiencias internacionales. Mirada Legislativa. No. 186. Obtenido de: http://bibliodigitalibd.senado.gob.mx/bitstream/handle/123456789/4882/ML_186.pdf?seq $\underline{\text { uence }=1 \& \text { is Allowed }=y}$ 
Ayala, H. (2009). Turismo cultural y consumo cultural en viajes turísticos. Cuba y el Centro Histórico de la Habana. Economía y Desarrollo, 154-185. Obtenido de https://www.redalyc.org/pdf/4255/425541313008.pdf

Bezerra, É. D., Conceicao, M., y Rocha, L. (2012). El turismo en la sociedad de la información: Un abordaje conceptual sobre el pos-turismo. Estudios y perspectivas en turismo, 5(21), 1262 - 1680. Recuperado el 16 de Junio de 2018, de http://www.scielo.org.ar/scielo.php?script=sci_arttext\&pid=S1851$17322012000500011 \& \operatorname{lng}=\mathrm{es} \& \mathrm{nrm}=\mathrm{iso} \& \ln \mathrm{ln}=\mathrm{es}$

Cantero, P., Escalera, J., García del Villar, R., y Hernández, M. (2000). Usos sociales del espacio público en el casco histórico de Sevilla. Zainak (19), 125 -140. Obtenido de https://core.ac.uk/download/pdf/11497963.pdf

Cantillo, J. (27 de agosto de 2020) Colombia lanzó un plan para reactivar el turismo en el país desde la semana próxima. Infobae. Obtenido de: https://www.infobae.com/america/colombia/2020/08/27/colombia-lanzo-un-plan-parareactivar-con-fuerza-el-turismo-en-todo-el-pais-desde-la-semana-proxima/

Carrión, F. (2005). El centro histórico como proyecto y objeto de deseo. EURE, XXXI, 15-35. Obtenido de http://www.redalyc.org:9081/home.oa?cid=6837631

Cazar, G. (2017). Tesis de Grado. Análisis Histórico de la Evolución Turística en el Barrio la Ronda (2006 - 2010). Quito, Pichincha, Ecuador: Universidad de las Américas.

Cazau, P. (2006). Introducción a la investigación en Ciencias Sociales. Buenos Aires: Tercera Edición. Obtenido de http://alcazaba.unex.es/asg/400758/MATERIALES/INTRODUCCI\%C3\%93N\%20A\%20 LA\%20INVESTIGACI\%C3\%93N\%20EN\%20CC.SS.pdf

Chateloin, F. (2008). El Centro Histórico ¿Concepto o criterio en desarrollo? Cujae: Revista de Arquitectura y Urbanismo, 10-23. Obtenido de: https://www.redalyc.org/pdf/3768/376839855003.pdf

Checa, M. (15 de marzo de 2010). Gentrificación y Cultura: Algunas reflexiones. Revista Bibliográfica de Geografía y Ciencias Sociales, XVI (914), 1 - 27. Obtenido de: https://www.researchgate.net/publication/215518724_Gentrificacion_y_cultura_algunas_ reflexiones

Corporación de Turismo Cartagena de Indias (2020) Plan Sectorial de Turismo de Cartagena de Indias, 2020-2023. Obtenido: https://www.cartagena.gov.co/Documentos/2020/Transparencia/TyALaIP/Planeacion/Pla nesDeDesarrollo/2020-2023/Anexo\%20Plan\%20Sectorial\%20de\%20Turismo.pdf

Crespi, M., y Domínguez, M. (2013). Urban strategies in tourist places. Cases of Madrid and Barcelona. Ocio y Turismo, 13-33. Obtenido de https://www.ucm.es/data/cont/media/www/pag92359/Crespi\%20Vallbona,\%20Dom\%C3\%ADnguez\%20P\%C3\%A9rez_2013_Urban\% 20strategies\%20in\%20tourist\%20places.\%20Cases\%20of\%20Madrid\%20and\%20Barcel ona.pdf

Crespi Vallbona, M., y Mascarilla Miró, Ó. (2018). La transformación y gentrificación turística del espacio urbano. Eure, 165 - 184. Obtenido de: https://scielo.conicyt.cl/scielo.php?script=sci_arttext\&pid=S0250-71612018000300051

Durán, L. (2014). La Ronda: Olvidar el barrio, recordar la calle. Quito: Flacso. Obtenido de http://repositorio.flacsoandes.edu.ec/xmlui/bitstream/handle/10469/6120/TFLACSO2014LFDS.pdf?sequence $=2 \&$ isAllowed $=y$ 
El Comercio (22 de mayo del 2020) Sector turístico de Ecuador perderá hasta USD 400 millones mensuales por la pandemia. Obtenido de:

https://www.elcomercio.com/tendencias/perdidas-sector-turistico-ecuadorcoronavirus.html

El Salto (4 de junio de 2020) Entrevista de Pedro Ramiro y Erika González a Ivan Murray “El turismo global es el Lehman Brothers de la crisis del COVID-19" Obtenido de: http://omal.info/spip.php?article9200

Flores, D., y De la O Barroso, M. (2012). La demanda turística internacional. Medio siglo de evolución. Revista de Economía Mundial, 1 - 22. Obtenido de: https://www.redalyc.org/pdf/866/86625395006.pdf

Frutos, Lopez -Roig, Serra Cobo y Devaux (12 de Mayo de 2020) COVID-19: The Conjunction of Events Leading to the Coronavirus Pandemic and Lessons to Learn for Future Threats. Frontiers in Medicine, 223. https://doi.org/10.3389/fmed.2020.00223

Gobierno de México (22 de junio del 2020) Guía para el turista internacional al llegar a México. Obtenido de: https://www.gob.mx/sectur/articulos/guia-para-el-turista-internacional-alllegar-a-mexico?idiom=es

Gómez, M., Galeano, C., y Jaramillo, D. (2015). El Estado del Arte: Una Metodología de Investigación. Revista Colombiana de Ciencias Sociales, 423 - 442.

Guerra Luzuriaga, P., (2019) Revisión de literatura en torno a la turistificación en espacios históricos - patrimoniales. Tesis de Grado. Universidad UTE. Quito, Ecuador. Obtenido de:

https://app.ute.edu.ec/sict/Biblioteca/libros.aspx?sq=13406611813121524407710612415 311300400221513706915504810918008624317713617414603800805003517517714711 424005006425301419416000703107204023316009706006102602914905418609321021 818013122801600222212509015324817420221200806408917209525009911004523921 $1125248027043178036232062139144088031201145 \&$ tpoTit $=064210163005051031046$ $239187039202011108100078006 \&$ catalogo $=17016313000307907510014708706503909$ $0187051112234 \&$ tip $=107163218125254205216196054116130153004044136173 \&$ cAso $\mathrm{c}=014034189182157190033114027239047244147119067069$

Hernández Sampieri, R., Fernández Collado, C., \& Baptista Lucio, P. (2014). Metodología de la Investigación. México D.F: McGraw Hill.

Hiernaux, D., y González, C. (2014). Gentrificación simbólica y poder en los centros históricos: Querétaro, México. Revista Electrónica de Geografía y Ciencias Sociales, 12(493), 1-14. Obtenido de http://revistes.ub.edu/index.php/ScriptaNova/article/view/15001

Hiernaux, D., y González, C. (2015). Patrimonio y turismo en centros históricos de ciudades medias. ¿Imaginarios encontrados? Revista de Estudios Urbanos y Ciencias Sociales, 5(2), 111 - 125. Obtenido de: https://dialnet.unirioja.es/servlet/articulo?codigo $=5372718$

Hosteltur (16 de septiembre de 2020). OMT: el turismo internacional puede caer hasta un $78 \%$ este año. Hosteltur. Obtenido de: https://www.hosteltur.com/139280_omt-el-turismointernacional-puede-caer-hasta-un-78-este-ano.html

Huete, R., y Mantecón, A. (2018). El auge de la turismofobia ¿hipótesis de investigación o ruido ideológico? Pasos Revista de Turismo y Patrimonio Cultural, 16(1), 9-19. Obtenido de http://www.pasosonline.org/Publicados/16118/PS118_01.pdf

Hurtado León, I., y Toro Garrido, J. (2007). Paradigmas y métodos de investigación en tiempos de cambio. Caracas: CEC.SA. 
Janoschka, M., Sequera, J., y Salinas, L. (2014). Gentrificación en España y América Latina. Revista de Geografía Norte Grande, 7-40. Obtenido de: https://scielo.conicyt.cl/scielo.php?script=sci_arttext\&pid=S0718-34022014000200002

La Moncloa (18 de junio de 2020) El Gobierno fortalece el turismo con un Plan estratégico de 4.262 millones de euros. Obtenido de: https://www.lamoncloa.gob.es/presidente/actividades/Paginas/2020/180620sanchezturismo.aspx

Martínez, P. (20 de julio del 2006) El método de estudio de caso: estrategia metodológica de la investigación científica. Pensamiento \& Gestión, núm.20.165-193. Universidad del Norte. Barranquilla Colombia. https://www.redalyc.org/pdf/646/64602005.pdf

Mena D. y Talxcala Q. (11 de agosto de 2020) PRI presenta propuestas para reactivar el turismo en México. Obtenido de: https://tlaxcala.quadratin.com.mx/principal/presenta-pripropuestas-para-reactivar-el-turismo-en-mexico/

Milano, C. (2018). Overtourism, malestar social y turismofobia. Pasos Revista de Turismo y Patrimonio Cultural, 16(3), 551-564. Obtenido de http://www.pasosonline.org/Publicados/16318/PS318_01.pdf

Ministerio de Turismo-MINTUR (17 de septiembre del 2020) El ministerio de turismo entregó el sello que reconoce a la capital del ecuador como un destino seguro. Obtenido de: https://www.turismo.gob.ec/el-ministerio-de-turismo-entrego-el-sello-que-reconoce-a-lacapital-del-ecuador-como-un-destino-seguro/

Molina, S. (2006). Posturismo: Turismo y posmodernidad. México: Trillas.

Navarrete, D. (2017). Turismo gentrificador en ciudades patrimoniales. Exclusión y transformaciones urbano-arquitectónicas del patrimonio en Guanajuato, México. INVI, 32(89), 61 -83. doi: http://dx.doi.org/10.4067/S0718-83582017000100061

Novelli, M. (18 de Julio de 2018). Overtourism: a growing global problem. The Conversation, págs. http://theconversation.com/overtourism-a-growing-global-problem-100029.

Onghena, Y., y Milano, C. (2015). Certezas e incertezas del turismo: movilidad y encuentro. Notes Internacionals, 1-5. Obtenido de https://www.cidob.org/es/publicaciones/serie_de_publicacion/notes_internacionals/n1_11 6_certezas_e_incertezas_del_turismo_movilidad_y_encuentro/certezas_e_incertezas_del _turismo_movilidad_y_encuentro

Organización Mundial del Turismo. (2016). Red de Turismo Urbano de la OMT. Plan de Acción. Obtenido de Organización Mundial del Turismo: http://cf.cdn.unwto.org/sites/all/files/docpdf/unwtocitytourismnetworkactionplanesweb.p df

Portal Oficial de Turismo de España (2020) COVID-19: Medidas para garantizar la seguridad de los turistas. Spain Info. Obtenido de: https://www.spain.info/es/descubrirespana/informacion-practica-turistas-covid-19-viajar-espana/

Quito Turismo. (diciembre de 2018). Quito en Cifras. Obtenido de Quito Turismo: https://www.quito-turismo.gob.ec/estadisticas/datos-turisticos-principales/category/82quito-en-cifras

Quito Turismo. (2020) Plan Emergente para la Reactivación del Sector Turístico del Distrito Metropolitano de Quito. Obtenido de: http://www.quitoturismo.gob.ec/descargas/LOTAIP2020/web/Plan\%20Emergente\%20Tur\%C3\%ADstico \%20del\%20DMQ.pdf 
Rius-Ulldemolins, J., y Posso, L. (2016). Cultura, transformación urbana y empoderamiento ciudadano frente a la gentrificación. Comparación entre el caso de Getsemaní (Cartagena de Indias) y el Raval (Barcelona). Revista EURE (Santiago), 22 - 34. Obtenido de https://scielo.conicyt.cl/pdf/eure/v42n126/art05.pdf

Rodríguez Aloma, P. (2008). El Centro Histórico: del concepto a la acción integral. Organización Latinoamericana y del Caribe de Centros Históricos, 51 - 64. Obtenido de http://www.redalyc.org/articulo.oa?id=115112534005

Rodríguez, J. (2009). Turismo urbano y sistema territorial. Almería, modelo emergente. Nimbus, 23-24, 171-181. Obtenido de https://dialnet.unirioja.es/descarga/articulo/3126870.pdf

Salgado, M. (2008). El Patrimonio Cultural como narrativa totalizadora y técnica de gubernamentalidad. Revista de la Organización Latinoamericana y del Caribe de Centros Históricos (1), 13-25. Obtenido de https://biblio.flacsoandes.edu.ec/catalog/resGet.php?resId=11206

Sequera y Nofre (2018) Shaken, not stirred: New debates on touristification and the limits of gentrification. Informa UK -Taylor \& Francis Group. Obtenido de: https://www.researchgate.net/publication/329249315_Shaken_not_stirred_New_debates on_touristification_and the limits_of_gentrification Doi: https://doi.org/10.1080/13604813.2018.1548819

Soto Vivar, A. P., y Soto, A. P. (2016). Espacios Públicos Patrimoniales: Continuidades históricas, culturales y paisajísticas entre zonas típicas y su entorno. Tesis de Posgrado. Chile: Pontificia Universidad Católica de Valparaíso. Obtenido de opac.pucv.cl/pucv_txt/txt-7500/UCD7695_01.pdf

Such Devessa M., Ramon A., Aranda P. (abril 29 de 2020) Turismo en tiempos de COVID. De la masificación al distanciamiento social. The conversation. Obtenido de: https://theconversation.com/turismo-en-tiempos-de-covid-19-de-la-masificacion-aldistanciamiento-social-137567 Abril 29 de 2020.

Tardivo, G., y Fernández, M. (2017). El uso del método histórico - comparativo en el análisis del liderazgo político: los casos de Bettino Craxi y Felipe González. Sociología Histórica, 291-318.

The Ostelea School of Tourism and Hospitality. (2017). Overtourism y turismofobia: Tendencias globales y contextos locales. Barcelona: The Ostelea School of Tourism and Hospitality.

World Tourism Organization (20 de enero del 2020a) International tourism growth continues to outpace the global economy. Obtenido de: https://www.unwto.org/international-tourismgrowth-continues-to-outpace-the-economy

World Tourism Organization (1 de Abril del 2020c) Call for Action for Tourism COVID-19 Mitigation and Recovery. Obtenido de: https://webunwto.s3.eu-west1.amazonaws.com/s3fs-public/2020-05/COVID-19-Tourism-Recovery-TAPackage_8\%20May-2020.pdf

World Tourism Organization (Mayo de 2020b) UNWTO World Tourism Barometer May 2020. Special Focus on the impact of COVID-19. Obtenido de: https://webunwto.s3.eu-west1.amazonaws.com/s3fs-public/2020-05/Barometer\%20-\%20May\%202020\%20$\% 20$ Short.pdf

Xian Liang, Z., y Gang Bao, J. (2014). Tourism gentrification in Shenzhen, China: Causes and socio spatial consequences. Tourism Geographies, 461 - 481. Obtenido de: https://www.researchgate.net/publication/272386569_Tourism_gentrification_in_Shenzh en_China_causes_and_socio-spatial_consequences 